\title{
LEISHMANIOSIS TEGUMENTARIA, UNA MIRADA A UNA "ENFERMEDAD TROPICAL DESATENDIDA"
}

\section{TEGUMENTARY LEISHMANIOSIS, A LOOK AT A NEGLECTED TROPICAL DISEASE}

\author{
Aidé Sandoval ${ }^{1, a}$, Gloria Minaya ${ }^{1, b}$
}

La Leishmaniosis es una protozoonosis endémica en el Perú, causada por parásitos del género Leishmania, se encuentra ampliamente extendida en el mundo, afectando a más de 12 millones de personas en todos los continentes, excepto Australia y la Antártida. La OMS estima que hay aproximadamente de 1 a 2 millones de casos nuevos anuales de Leishmaniosis a nivel mundial (1). En la mayoría de países latinoamericanos constituye un problema de salud pública de gran importancia, no solo a causa del incremento del número de casos en diversas latitudes, sino en particular por el impacto socioeconómico y psicológico, característico de esta enfermedad, que con frecuencia es mutilante ${ }^{(2)}$.

Su distribución geográfica está determinada por factores ecológicos reguladores de la presencia de los insectos vectores pertenecientes a diferentes especies del género Lutzomyia (fllebotomos), conocidos como "titiras"o "manta blanca" y la existencia de los todavía no bien conocidos reservorios naturales ${ }^{(3)}$.

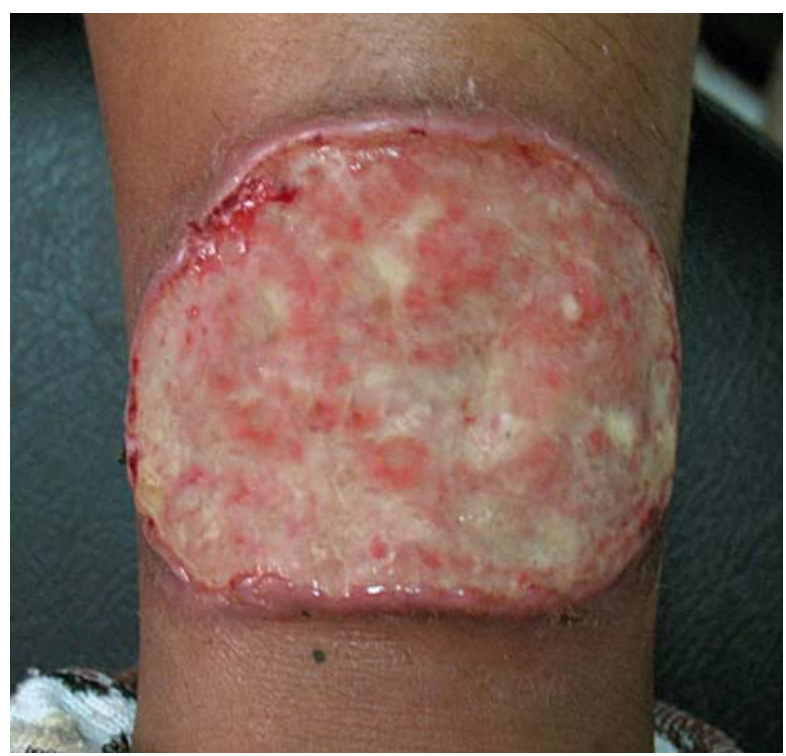

Foto 1. Leishmaniosis selvática cutánea, con marcada inflamación en pierna izquierda.

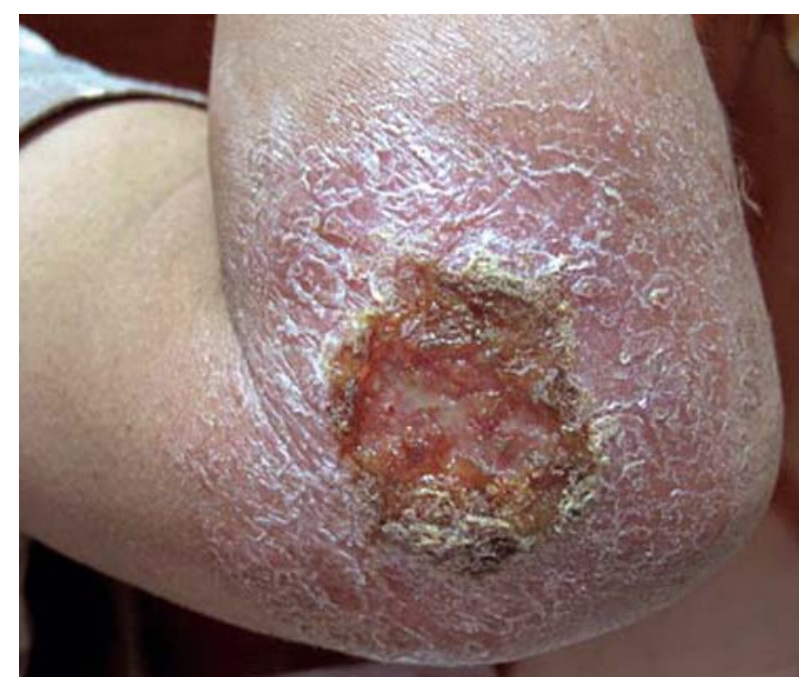

Foto 2. Leishmaniosis andina cutánea, úlcerocostrosa con proceso inflamatorio eczematizado.

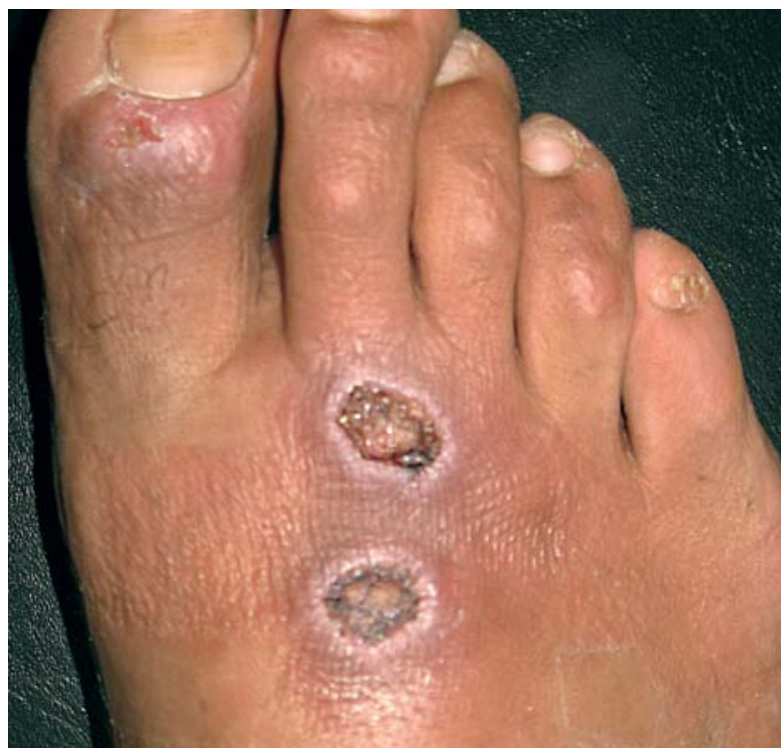

Foto 3. Leishmaniosis selvática cutánea, lesión ulcerativa múltiple.

Laboratorio de Leishmaniosis y Chagas, Instituto Nacional de Salud. Lima, Perú.

a Bióloga; ${ }^{b}$ Bióloga, Máster en Medicina Tropical y Salud Internacional

Recibido: 23-09-11 Aprobado: 24-09-11 
En el Perú, la enfermedad se presenta desde épocas precolombinas, en focos endémicos. Las formas clínicas predominantes, de acuerdo con las características clínicas y geográficas, son la Leishmaniosis cutáneo andina, forma benigna llamada "uta", la cual deja cicatrices características, que está presente en la vertiente occidental de los andes y valles interandinos entre niveles altitudinales que van desde los 1200 a los $3000 \mathrm{~m}$ de altitud, y la Leishmaniosis cutáneo-mucosa selvática, que es metastásica, cuya forma maligna es conocida como "espundia", de evolución crónica y

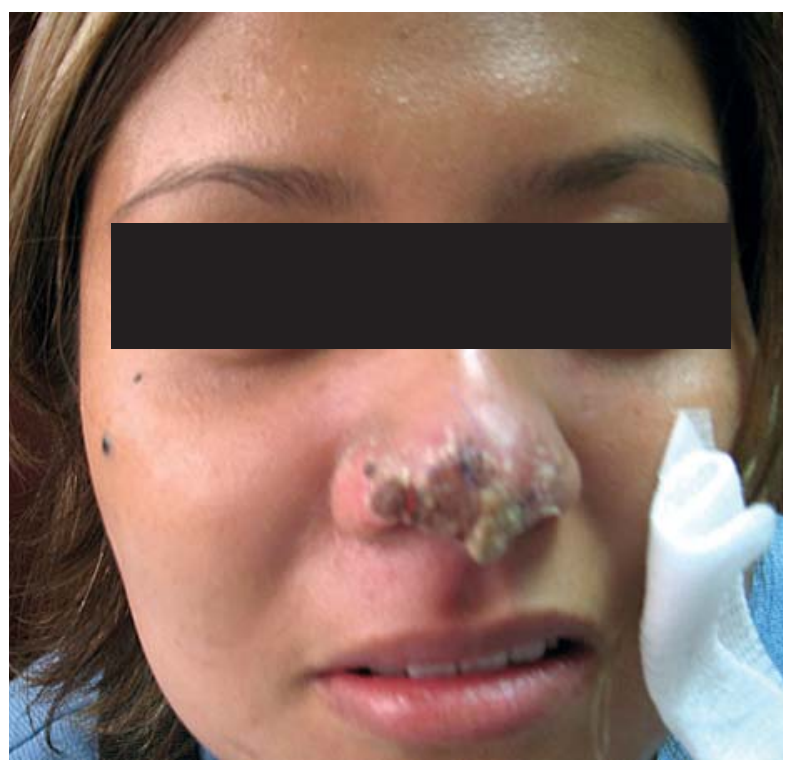

Foto 4. Leishmaniosis andina cutáneo-mucosa, lesión úlcerocostrosa en pirámide nasal deformante.

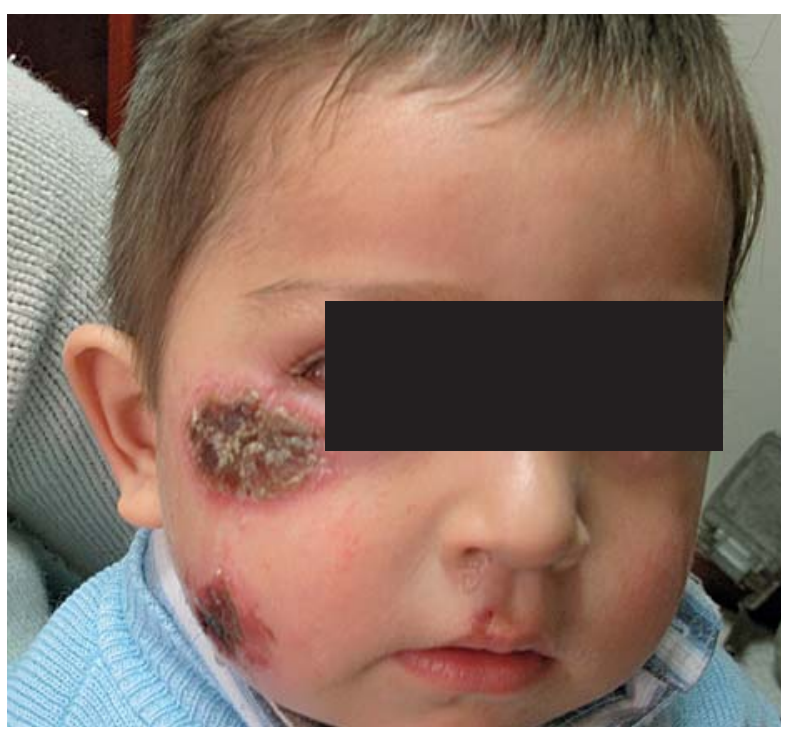

Foto 5. Leishmaniosis selvática cutánea, úlcerocostrosa en cara. de difícil control terapéutico, distribuida en la vertiente oriental andina, desde los $900 \mathrm{~m}$ de altitud, a través de todo el llano amazónico selvático, aunque en algunas regiones podrían confluir ambas formas clínicas ${ }^{(4,5)}$.

Se han descrito también algunos casos de Leishmaniosis cutánea difusa, forma anérgica de la enfermedad, procedentes de la selva central del Perú ${ }^{(6,7)}$. En esta galería se presentan fotografías de algunos pacientes derivados al Instituto Nacional de Salud para su confirmación diagnóstica.

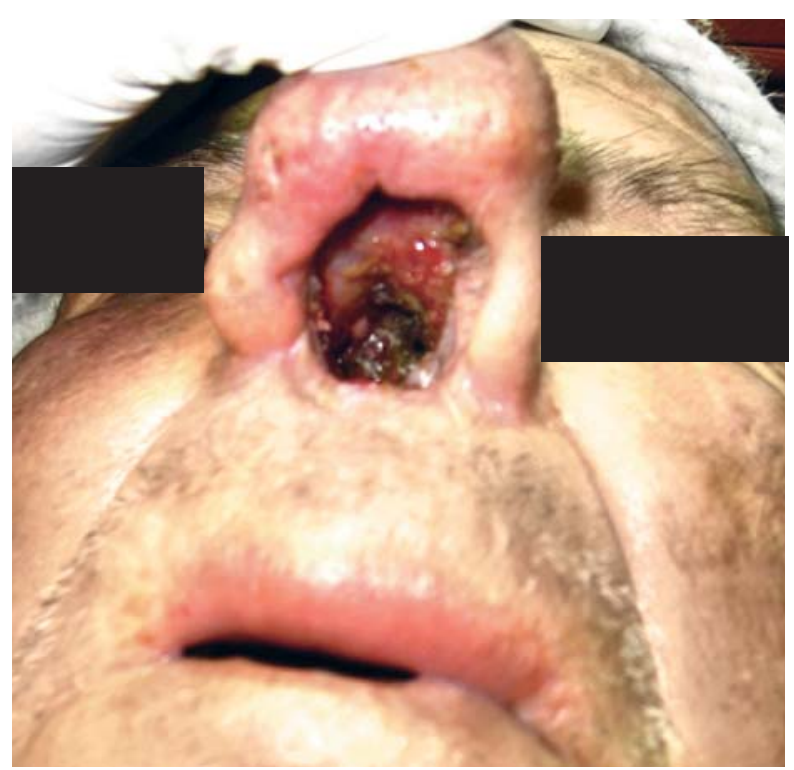

Foto 6. Leishmaniosis selvática cutáneo-mucosa tardía con destrucción de tabique nasal.

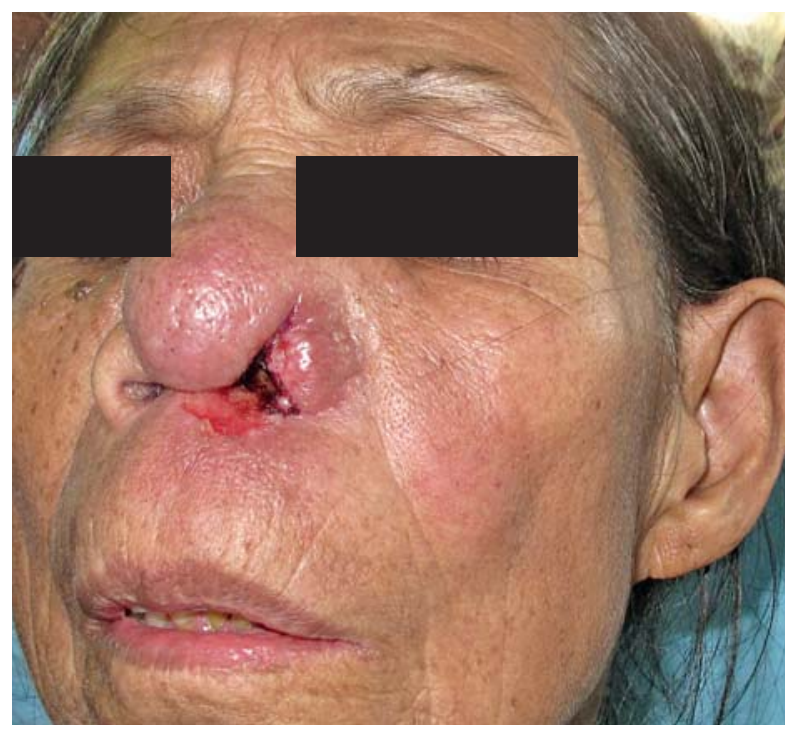

Foto 7. Leishmaniosis selvática cutáneo-mucosa tardía, "nariz de tapir". 


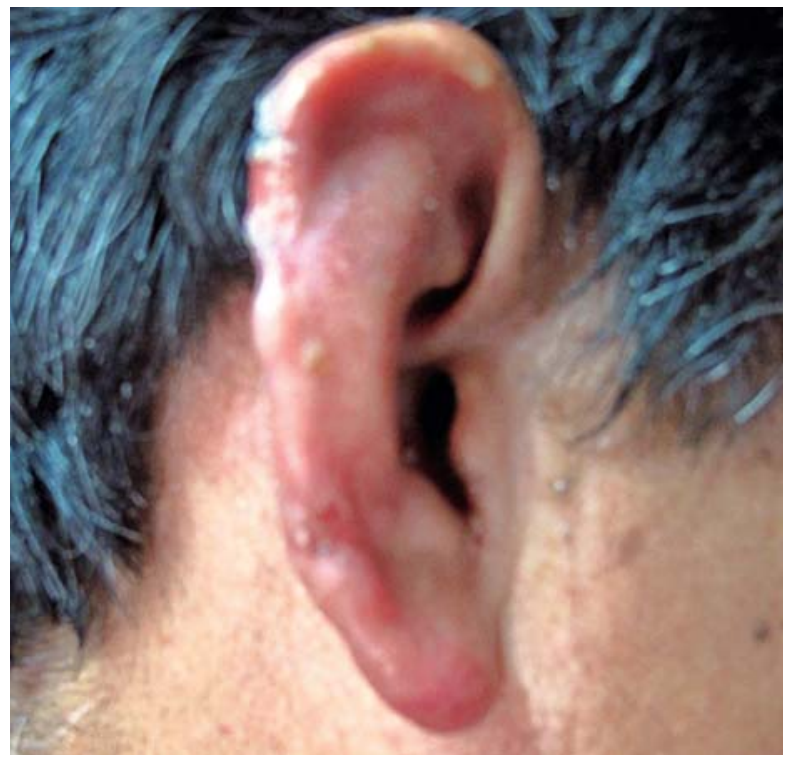

Foto 8. Leishmaniosis andina cutánea tardía, de pabellón auricular.

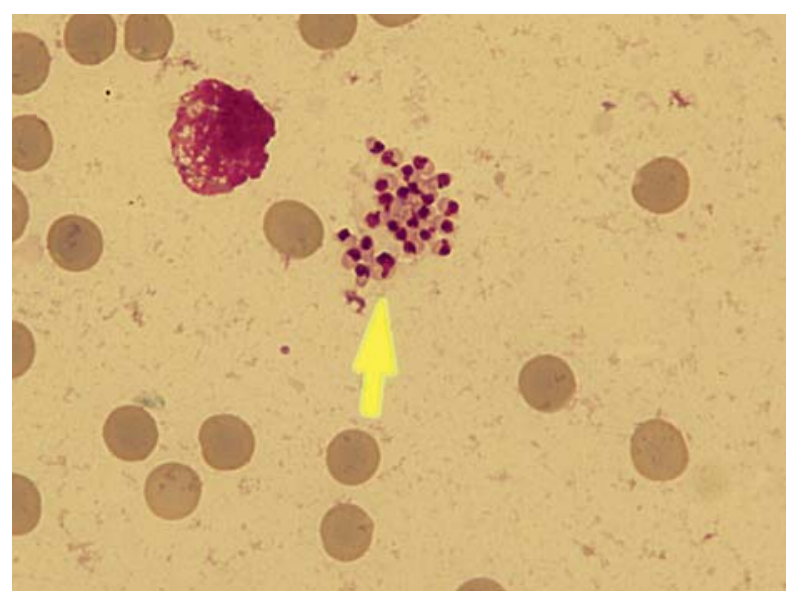

Foto 9. Forma amastigota del parásito, se observa en lámina de frotis de lesiones obtenidas por raspado, aspirado o impronta de biopsias de tejido, coloreado con Giemsa, 1000x.

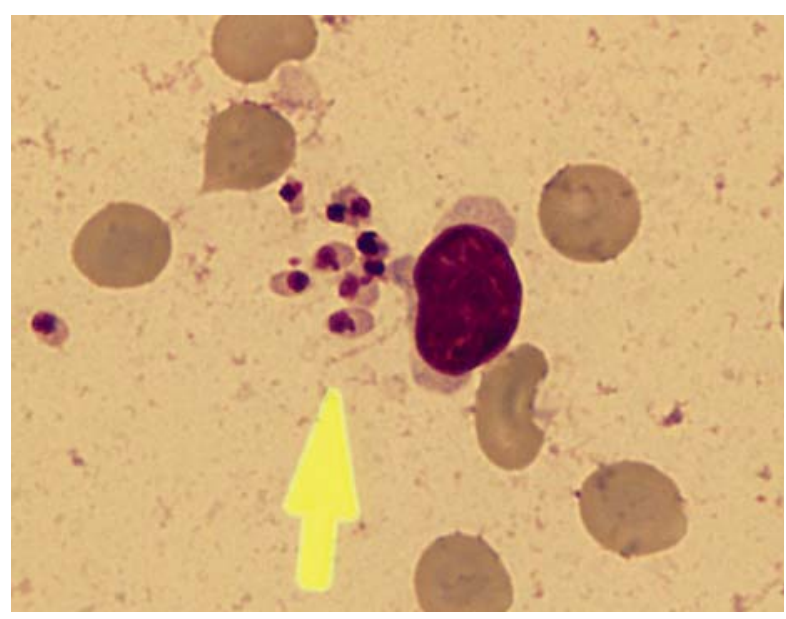

Foto 10. Forma amastigota del parásito en frotis coloreado con Giemsa, observación microscópica a 1000x.

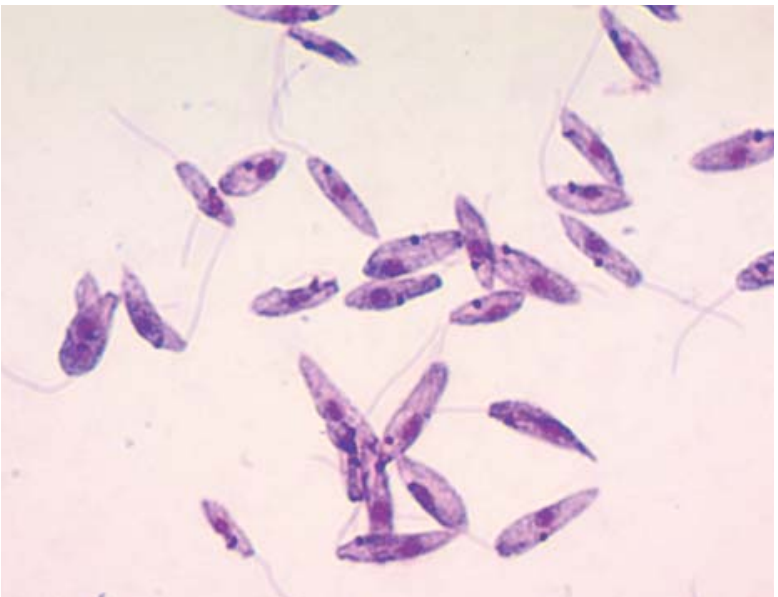

Foto 11. Forma promastigota, se observa en cultivos de raspado, aspirado y biopsias de lesiones.

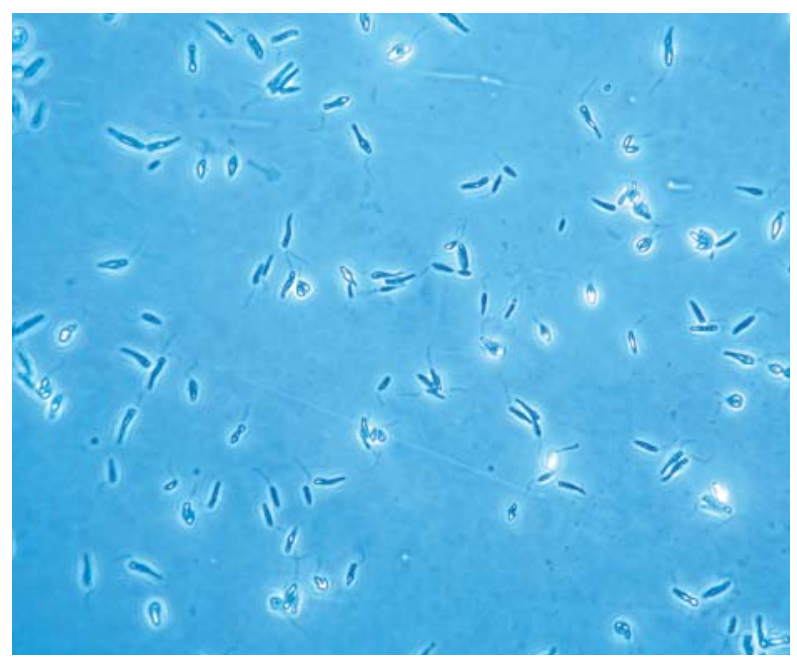

Foto 12. Forma promastigota móvil en cultivo in vitro, observación al microscopio de contraste de fase, 400x.

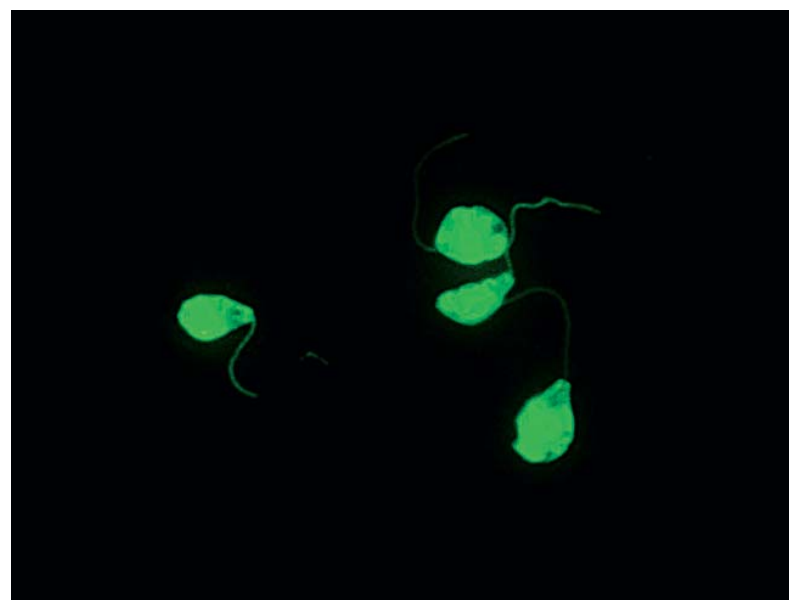

Foto 13. Observación microscópica del parásito mediante la técnica de inmunofluorescencia indirecta, 1000x . 


\section{REFERENCIAS BIBLIOGRÁFICAS}

1. Kenner JR, Aronson EN, Benson PM. Advances in Military Dermatology: The United States Military and Leishmaniasis. En: Aronson EN. Dermatologic Clinics. 1ra ed. Philadelphia: W.B. Saunders Company; 1999. p. 77-91.

2. Valverde J, Vicuña D. Aspectos clínicos, epidemiológicos e histológicos de la leishmaniasis cutánea andina. Folia Dermatol. 2003;14(3):10-4.

3. Neyra D. Las leishmaniasis en el Perú. Folia Dermatol. 1997;8(2):51-5.

4. Miranda H. Andean cutaneous leishmaniasis in the highlands of the Department of La Libertad in Peru. En: Research on Control Strategies for the Leishmaniases. Proceedings of an International Workshop held in Otawa, Canada. IDRV/CRDI/CIID; 1987. p.148-49.

5. Burstein Z, Guillén A. Leishmaniasis cutánea y mucocutánea (CIE - 10 B55 1 - B55 - 2) en el Perú. Rev Peru Med Exp Salud Publica. 2002;19(4):215-8.
6. Franke ED, Lucas CM, Tovar AA, Kruger JH, De Rivera MV, Wignall FS. Diffuse cutaneous leishmaniasis acquired in Peru. Am J Trop Med Hyg. 1990;43(3):260-2.

7. Lucas CM, Franke ED, Cachay MI, Tejada A, Cruz ME, Kreutzer RD, et al. Geographic distribution and clinical description of leishmaniasis cases in Peru. Am J Trop Med Hyg. 1998;59(2):312-7.
Correspondencia: Aidé Sandoval.

Dirección: Laboratorio Leishmaniosis y Chagas, Instituto Nacional Salud, Cápac Yupanqui 1400. Lima, Perú.

Teléfono: (511) 6176200 - 2167

Correo electrónico: asandoval@ins.gob.pe

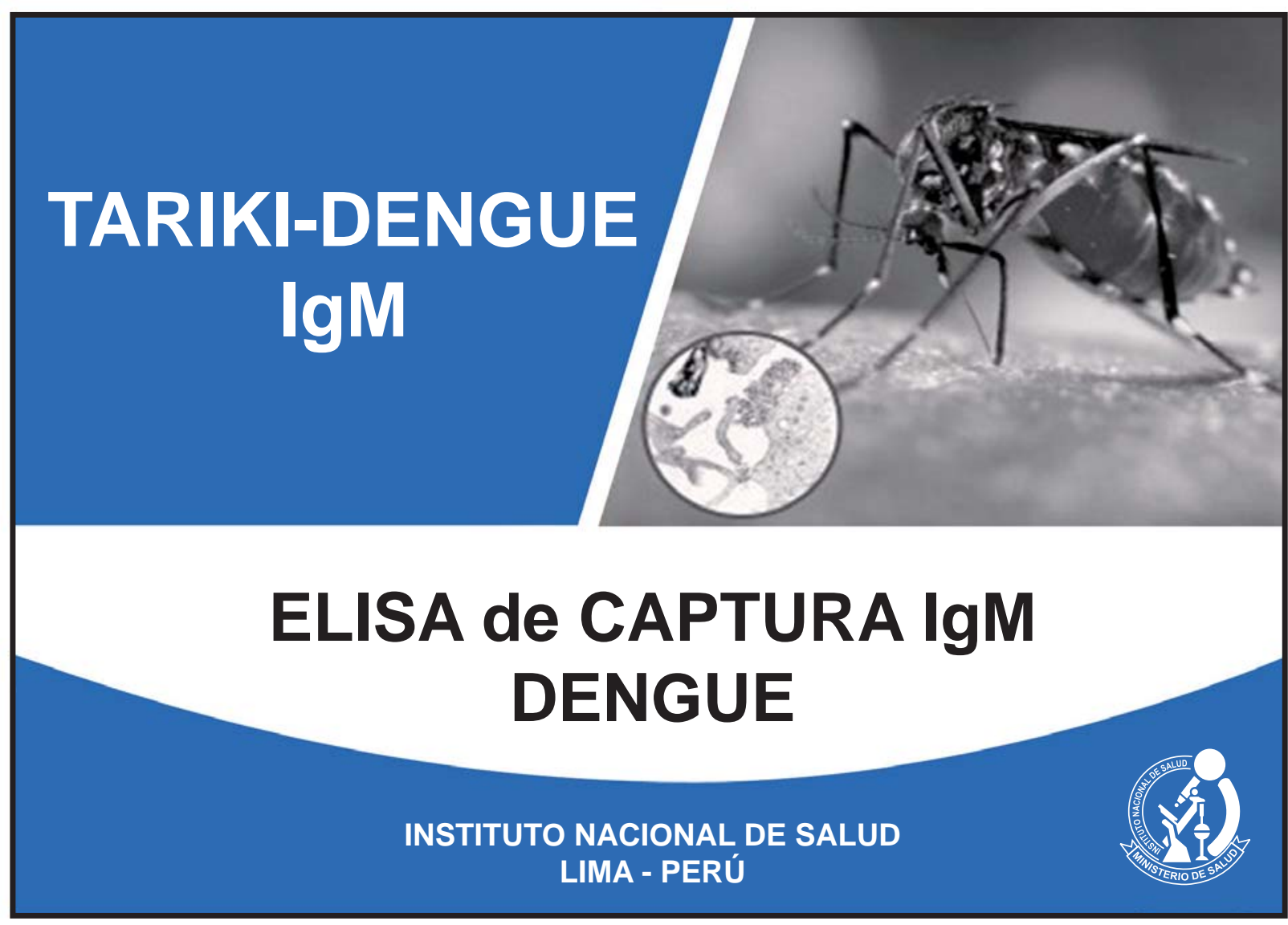

\title{
Pronto disponible en el Perú....
}

\author{
Kit para la determinación de anticuerpos IgM anti Dengue, \\ desarrollado y producido por el Instituto Nacional de Salud. \\ INS/MINSA
}

